\title{
Variable Protease-Sensitive Prionopathy Transmission to Bank Voles
}

\author{
Romolo Nonno, ${ }^{1}$ Silvio Notari, ${ }^{1}$ Michele Angelo Di Bari, Ignazio Cali, \\ Laura Pirisinu, Claudia d'Agostino, Laura Cracco, Diane Kofskey, Ilaria Vanni, \\ Jody Lavrich, Piero Parchi, Umberto Agrimi, Pierluigi Gambetti
}

\begin{abstract}
Variably protease-sensitive prionopathy (VPSPr), a recently described human sporadic prion disease, features a protease-resistant, disease-related prion protein (resPrPD) displaying 5 fragments reminiscent of Gerstmann-SträusslerScheinker disease. Experimental VPSPr transmission to human PrP-expressing transgenic mice, although replication of the VPSPr resPrPD profile succeeded, has been incomplete because of second passage failure. We bioassayed VPSPr in bank voles, which are susceptible to human prion strains. Transmission was complete; first-passage attack rates were $5 \%-35 \%$, and second-passage rates reached $100 \%$ and survival times were $50 \%$ shorter. We observed 3 distinct phenotypes and resPrPD profiles; 2 imitated sporadic Creutzfeldt-Jakob disease resPrP ${ }^{\mathrm{D}}$, and 1 resembled Gerstmann-Sträussler-Scheinker disease resPrPD. The first 2 phenotypes may be related to the presence of minor PrPD components in VPSPr. Full VPSPr transmission confirms permissiveness of bank voles to human prions and suggests that bank vole PrP may efficiently reveal an underrepresented native strain but does not replicate the complex VPSPr PrPD profile.
\end{abstract}

Sporadic prion diseases are classified according to phenotype as well as the pairing of the prion protein (PrP) genotype at the methionine $(\mathrm{M})$ /valine $(\mathrm{V})$ polymorphic codon 129 and the conformational characteristics of the abnormal or disease-associated $\operatorname{PrP}\left(\mathrm{PrP}^{\mathrm{D}}\right)$. These characteristics include electrophoretic mobility and the ratio of the $\operatorname{PrP}^{\mathrm{D}}$ fragments that are resistant to proteinase $\mathrm{K}(\mathrm{PK})$ digestion (Appendix Table 1, https://wwwnc.cdc.gov/EID/article/25/1/18-0807App1.pdf) (1). According to these criteria, the 3 major types of sporadic prion disease are sporadic Creutzfeldt-Jakob

Author affiliations: Istituto Superiore di Sanità, Rome, Italy

(R. Nonno, M.A. Di Bari, L. Pirisinu, C. d'Agostino, I. Vanni, U. Agrimi); Case Western Reserve University, Cleveland, Ohio,

USA (S. Notari, I. Cali, L. Cracco, D. Kofskey, J. Lavrich, P. Gambetti); University of Bologna, Bologna, Italy (P. Parchi); Istituto delle Scienze Neurologiche di Bologna (P. Parchi)

DOI: https://doi.org/10.3201/eid2501.180807 disease (sCJD), sporadic fatal insomnia, and variably protease-sensitive prionopathy (VPSPr) (2-5).

VPSPr was first reported in 2008 and further defined in $2010(6-8)$ as a sporadic prion disease distinct from SCJD. Since then, 37 cases have been reported, consistent with a prevalence rate of $1 \%-2 \%$ for all sporadic prion diseases (8). Similar to SCJD, VPSPr targets all 3 PrP genotypes. However, the prevalence of the 3 genotypes at codon 129 (MM, MV, and VV) greatly differs, indeed is almost inverted, in the 2 diseases: homozygosity $\mathrm{VV}$ is the most common $(65 \%)$ genotype in VPSPr and the least common $(16 \%)$ in $\operatorname{sCJD}(2,9)$. Furthermore, at variance with SCJD, in which the 129 genotype is a determinant of disease phenotype and $\operatorname{PrP}^{\mathrm{D}}$ characteristics, the 129 genotype influence on phenotype, although present, is subtle $(3,7,8)$. These differences point to a distinct role of the 129 genotype as a risk factor and imply that the etiologic-pathogenetic mechanisms of the 2 diseases differ.

Although the histopathology of VPSPr is distinct (e.g., spongiform degeneration, frequent presence of PrP microplaques, and a recognizable $\operatorname{PrP}^{\mathrm{D}}$ immunostaining pattern), the hallmarks of VPSPr are the characteristics of its $\operatorname{PrP}^{\mathrm{D}}$. In contrast to virtually all other sporadic human prion diseases, in which PK-resistant $\operatorname{PrP}^{\mathrm{D}}$ (resPrP ${ }^{\mathrm{D}}$ ) electrophoretically separates into 3 major bands, VPSPr resPrP ${ }^{\mathrm{D}}$ characteristically separates into 5 bands. Furthermore, although the 3 bands of resPrP $\mathrm{P}^{\mathrm{D}}$ are all cleaved by $\mathrm{PK}$ exclusively at the $\mathrm{N}$ terminus and separate according to the presence of 2 , 1 , or 0 sugar moieties, VPSPr resPrP $\mathrm{P}^{\mathrm{D}}$ bands include only the monoglycosylated and unglycosylated forms, which are cleaved either only at the $\mathrm{N}$ terminus or at both the $\mathrm{N}$ - and $\mathrm{C}$-termini. Thus, the $\mathrm{C}$-terminus-truncated resPrP $\mathrm{P}^{\mathrm{D}}$ lacks the GPI (glycosylphosphatidylinositol) anchor. Additional variances concerning immunoreactivity characteristics, ratios of PK-resistant and $\mathrm{PK}$-sensitive $\operatorname{PrP}^{\mathrm{D}}$ species, and conformational properties including aggregate size, have also been observed (6-8). These distinctive properties point to VPSPr PrPD as a prion strain different from those of other

${ }^{1}$ These authors contributed equally to this article. 
sporadic prion diseases. However, the VPSPr prion shares the multiplicity of the resPrP ${ }^{\mathrm{D}}$ electrophoretic bands with prions from a subset of inherited prion diseases referred to as Gerstmann-Sträussler-Scheinker disease (GSS), prompting the suggestion that VPSPr is the sporadic form of $\operatorname{GSS}(7,10)$. Furthermore, the presence of small amounts of sCJD-like 3-band resPrP $\mathrm{P}^{\mathrm{D}}$ has also been signaled in $\operatorname{VPSPr}(6,11,12)$.

Disease transmission to receptive hosts is a valuable way to further define the characteristics of strains associated with prion diseases. VPSPr has been experimentally transmitted to 3 lines of transgenic mice expressing normal $\operatorname{PrP}$ or cellular human $\operatorname{PrP}\left(\operatorname{PrP}^{\mathrm{C}}\right)$, harboring residue $\mathrm{M}$, $\mathrm{V}$, or MV at residue $129(13,14)$. Data in all experiments were essentially similar. Inoculated mice remained asymptomatic, but half showed focal $\operatorname{PrP}^{\mathrm{D}}$ plaques with minimal spongiform degeneration, and $\operatorname{PrP}^{\mathrm{D}}$ mimicking the electrophoretic profile of the native $\operatorname{PrP}^{\mathrm{D}}$ on immunoblot was demonstrated in about one third of the inoculated mice. No transmission was observed at second passage.

The bank vole, a small rodent resembling the mouse with which it shares the entire sequence of normal $\operatorname{PrP}$ or $\operatorname{PrP}^{\mathrm{C}}$ except for 8 aa, but whose sequence differs from human PrPC by 15 aa, has recently emerged as a particularly permissive host. Bank voles and transgenic mice expressing bank vole $\operatorname{PrP}^{\mathrm{C}}$ have been successfully infected after challenge with human and animal prion diseases that are hard to transmit even to recipients expressing homologous $\operatorname{PrPC}^{\mathrm{C}}(15-18)$.

We studied transmission of VPSPr from patients with MM, MV, and VV codon 129 genotypes to bank voles harboring either the PrP genotype 109M (bv109M) or 109I (bv109I). Although the attack rate was generally low at first passage, it consistently raised to $100 \%$ at second passage, when survival times also decreased on average by $>50 \%$. We identified $3 \operatorname{PrP}^{\mathrm{D}}$ isoforms with the characteristics of distinct strains in the affected bank voles.

\section{Materials and Methods}

The inocula used in the first passage were brain homogenates from 7 persons with a definitive diagnosis of VPSPr: 2 with genotypes $129 \mathrm{MM}, 3$ with $129 \mathrm{MV}$, and 2 with $129 \mathrm{VV}$. Homogenate was inoculated into the cerebrum of 205 bank voles according to previously described procedures (16). The bank vole brains were processed for histopathology, immunohistochemistry, lesion profiles, and paraffin-embedded tissue (PET) blots according to previously reported procedures (15). Western blot was performed according to Notari et al. (19). The insoluble fraction was prepared according to previously described procedures (20). Preparation of monoclonal antibodies is described in the Appendix. Statistical significance was determined by 1-way analysis of variance, followed by the Tukey multiple comparison test.

\section{Results}

\section{Transmission Characteristics}

At first passage, attack rates of VPSPr were 35\% (29/82) in bv109I and 5\% (3/59) in bv109M (Table 1; Appendix Table 2). The 2 bank vole genotypes diverged as to disease transmission in 2 ways. First, all VPSPr 129 genotypes were transmitted to bv109I, but bv109M were not susceptible to VPSPr-VV. Second, bv109I propagated 3 distinct histopathologic phenotypes and matching $\operatorname{PrP}^{\mathrm{D}}$ types (hereafter identified as T1, T2, and T3), but bv109M replicated the T1 phenotype exclusively. A more detailed analysis in bv109I,

\begin{tabular}{|c|c|c|c|c|c|c|c|c|c|c|c|c|c|}
\hline \multirow{6}{*}{$\begin{array}{l}\text { Inoculum } \\
\text { VPSPr-MM, } \\
\mathrm{n}=2\end{array}$} & \multicolumn{7}{|c|}{ Bv109l } & \multicolumn{6}{|c|}{ Bv109M } \\
\hline & \multicolumn{3}{|c|}{ 1st passage } & \multicolumn{4}{|c|}{ 2nd passage } & \multicolumn{3}{|c|}{ 1st passage } & \multicolumn{3}{|c|}{ 2nd passage } \\
\hline & $\begin{array}{l}\mathrm{PrP}^{\mathrm{D}} \\
\text { type }\end{array}$ & $\begin{array}{c}\text { Attack } \\
\text { rate }\end{array}$ & $\begin{array}{l}\text { Survival } \\
\text { time, dpi } \\
\pm S D\end{array}$ & $\begin{array}{l}\mathrm{PrP}^{\mathrm{D}} \\
\text { type }\end{array}$ & $\begin{array}{c}\text { Attack } \\
\text { rate }\end{array}$ & $\begin{array}{c}\text { Survival } \\
\text { time, dpi } \\
\pm \text { SD }\end{array}$ & $\begin{array}{c}\text { Survival } \\
\text { reduction, } \\
\%\end{array}$ & $\begin{array}{l}\mathrm{PrP}^{\mathrm{D}} \\
\text { type }\end{array}$ & $\begin{array}{c}\text { Attack } \\
\text { rate }\end{array}$ & DPI & $\begin{array}{l}\mathrm{PrP}^{\mathrm{D}} \\
\text { type }\end{array}$ & $\begin{array}{c}\text { Attack } \\
\text { rate }\end{array}$ & $\begin{array}{c}\text { Survival } \\
\text { time, dpi } \\
\pm \mathrm{SD}\end{array}$ \\
\hline & T1 & 1/20† & 901 & NA & NA & NA & NA & T1 & $1 / 15$ & 356 & T1 & $11 / 11$ & $148 \pm 12$ \\
\hline & T2 & $1 / 20$ & 839 & NA & NA & NA & NA & & & & & & \\
\hline & T3 & $5 / 20$ & $\begin{array}{c}413 \pm \\
102\end{array}$ & T3 & $9 / 9$ & $\begin{array}{c}247 \pm \\
35 \ddagger\end{array}$ & 40 & & & & & & \\
\hline \multirow[t]{3}{*}{$\begin{array}{l}\text { VPSPr-MV, } \\
\mathrm{n}=3\end{array}$} & T1 & 13/44† & $\begin{array}{c}458 \pm \\
137\end{array}$ & T1 & $10 / 10$ & $\begin{array}{c}195 \pm \\
9 \S\end{array}$ & 57 & $\mathrm{~T} 1$ & $2 / 30$ & $\begin{array}{l}290 \\
588\end{array}$ & $\mathrm{~T} 1$ & $11 / 11$ & $142 \pm 11$ \\
\hline & T2 & $6 / 44$ & $\begin{array}{l}872 \pm \\
1109\end{array}$ & T2 & $14 / 14$ & $\begin{array}{l}338 \pm \\
100 \#\end{array}$ & 61 & & & & & & \\
\hline & T3 & $1 / 44$ & 554 & NA & NA & NA & NA & & & & & & \\
\hline $\begin{array}{l}\text { VPSPr-VV, } \\
\mathrm{n}=2\end{array}$ & T3 & $2 / 18$ & 596,535 & T3 & $9 / 9$ & $\begin{array}{c}255 \pm \\
24^{* *}\end{array}$ & 55 & NA & $0 / 14$ & NA & $\mathrm{NA}$ & NA & NA \\
\hline
\end{tabular}

*bv, bank voles; dpi, days postinoculation; NA, not available; neg, negative; PRP ${ }^{\mathrm{D}}$, disease-associated prion protein; VPSPr, variably protease-sensitive prionopathy.

†Total no. bank voles inoculated with either VPSPr-MM or VPSPr-MV; statistics of survival times at 1st vs. 2nd passages.

$\mp \mathrm{p}<0.05$.

$\S p<0.0001$.

TIncubation periods of T2 vs. T1 $\mathrm{p}<0.0001$

$\# p<0.0001$.

${ }^{* *} p<0.001$ 
Variable Protease-Sensitive Prionopathy

Table 2. Itemized VPSPr transmission features in bv109l at first passage, by phenotype*

\begin{tabular}{|c|c|c|c|c|c|c|c|}
\hline Genotype & \multicolumn{2}{|c|}{ T1 } & \multicolumn{2}{|c|}{ T2 } & \multicolumn{2}{|c|}{ T3 } & $\begin{array}{c}\text { Attack rate for } \\
\text { all } \\
\text { phenotypes, \% }\end{array}$ \\
\hline $\mathrm{MM}$ & 5 & 901 & 5 & 839 & 25 & $413 \pm 102 \dagger$ & 35 \\
\hline VV & 0 & NA & 0 & NA & 11.1 & 596,535 & 11.1 \\
\hline All affected genotypes & $11.5 \ddagger$ & $490 \pm 177 \dagger$ & $6.2 \ddagger$ & $867 \pm 101 \dagger$ & $12.8 \ddagger$ & $469 \pm 110 \dagger$ & $30.5 \ddagger$ \\
\hline
\end{tabular}

although limited by the low number of animals in each subset, suggested a lower attack rate for VPSPr-VV, the most common form of human VPSPr, compared with the MM and MV genotypes and a prevalence for T3 that was 11\% higher than that for T1 and 106\% higher than that for T2 (Table 2). Overall survival times were 575 days postinoculation (dpi) for bv109I and 411 dpi for bv109M. However, when we considered only the bank voles associated with the T1 phenotype, because bv109M were exclusively associated with T1, the dpi difference became smaller: 490 dpi for bv109I and $411 \mathrm{dpi}$ for bv109M (Tables 1, 2). As for survival times related to T1-T3 phenotypes and VPSPr genotypes, the survival times for $\mathrm{T} 2$ were nearly twice those for $\mathrm{T} 1$ and $\mathrm{T} 3$ (Table 2).

Second passage in bv109I was invariably characterized by a $100 \%$ attack rate, a $40 \%-61 \%$ decrease in survival times, and conservation of the original phenotype (Table 1). A similar trend was observed for bv109M.

\section{Histopathology and Immunohistochemistry}

Phenotype T1 featured finely vacuolated spongiform degeneration often involving the entire thickness of the neocortex, including the molecular layer but occasionally also showing a laminar distribution (Figure 1, https://wwwnc.cdc.gov/eid/ article/25/1/18-0807-f1). On second passage, the spongiform degeneration appeared to be more widespread, also affecting the hippocampus and subcortical structures such as basal nuclei, thalamus, and superior colliculi but not the cerebellum. PrP immunohistochemistry demonstrated punctate deposits often co-distributed with spongiform degeneration (Figure 1, column T1, row ii). At second passage, T1 features did not differ significantly between bv109M and bv109I. Furthermore, $\mathrm{T} 1$ also resembled the histopathologic phenotype shown by bv109M and bv109I after inoculation with SCJDMM1 or sCJDMV1, respectively (Figures 2, 3; Appendix Figure 1)

In phenotype $\mathrm{T} 2$, spongiform degeneration affected predominantly subcortical structures over neocortical regions, especially the hypothalamus with the apparent exclusion of the mammillary bodies (Figure 1, column T2, row iii). PrP immunohistochemistry showed granular deposits occasionally resembling miniature plaquelike formations rather than the punctate deposits of the T1 phenotype (Figure 1, column T2, row iv).
Phenotype T3 was characterized by the paucity of spongiform degeneration in the cerebral neocortex and subcortical gray matter structures; spongiform degeneration was often prominent in the regions of the hemispheric white matter lying above the hippocampus and in the corpus callosum, where parenchyma was occasionally disorganized with glial reaction. PrP immunostaining was mostly limited to those regions where it often aggregated in confluent plaque-like deposits but not well-formed plaques (Figure 1, column T3, row v, and column Tc, row vi). No remarkable differences were detected between first and second passages. Overall, the T3 histopathologic phenotype resembled that shown by bv109I after inoculation with brain homogenates from some GSS subtypes (16).

It is noteworthy that the T1-T3 phenotypes were never observed to coexist in 1 animal, although distinct phenotypes were often observed in bank voles receiving the same inoculum. Although all 3 phenotypes occurred after inoculation with VPSPr-MM or -MV, the sole phenotype associated with VPSPr-VV inoculation was T3 (Table 1).

\section{Lesion Profiles and PET Blots}

Profiles of spongiform degeneration as a function of lesion severity and brain anatomic location confirmed the distinctive characteristics of the T1-T3 phenotypes (Figure 2, panel A; Appendix Figure 1). The T1 spongiform degeneration profile in bv109I did not differ significantly from that of bv109M; both mirrored the profiles of bv109I inoculated with SCJDMV1 and bv109M inoculated with SCJDMM1 brain homogenate (Figure 2, panel B; Appendix Figure 1).

The PET blot patterns of brain $\operatorname{PrP}^{\mathrm{D}}$ distribution were also quite distinct in the 3 phenotypes and, overall, reproduced the spongiform degeneration distribution (Figures 2, 3). In $\mathrm{T} 1, \operatorname{PrP}^{\mathrm{D}}$ was well represented in selected regions including cerebral neocortex and hippocampus, basal nuclei, thalamus, superior colliculi, geniculate nuclei, and substantia nigra but not in the cerebellum and lower brain stem. No significant variations were detected between $\operatorname{PrP}^{\mathrm{D}}$ distributions at first and second passages (data not shown). $\operatorname{PrP}^{\mathrm{D}}$ distributions were also similar in bv109I and bv109M inoculated with classic sCJDMV1 and SCJDMM1 prions, respectively (Figure 3, panel B). In the $\mathrm{T} 2$ phenotype, $\operatorname{PrP}^{\mathrm{D}}$ appeared to be present in moderate and uniform amounts in several 

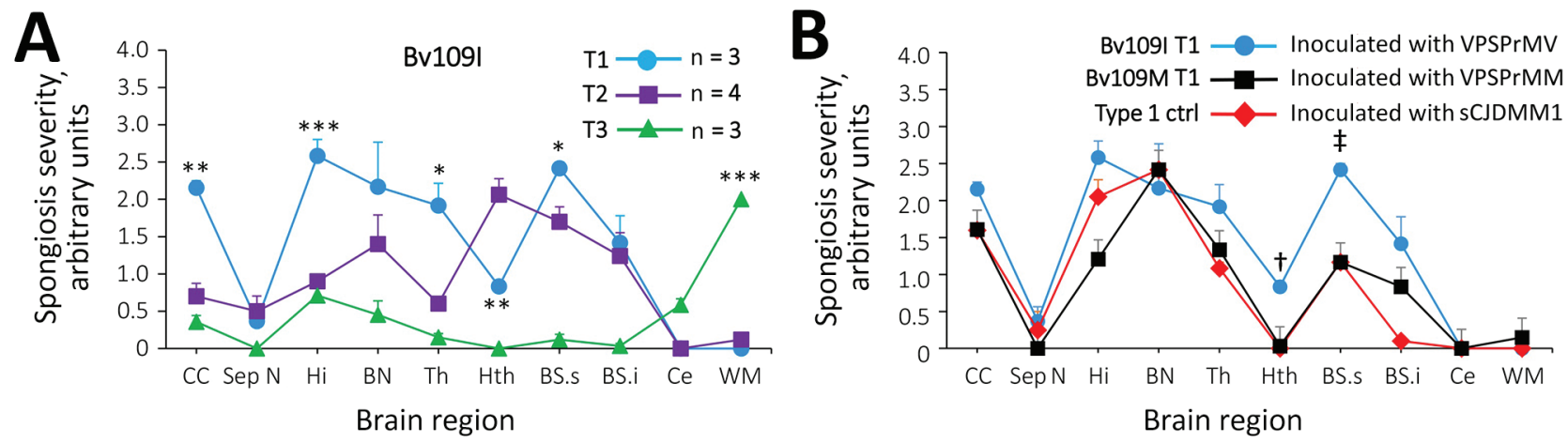

Figure 2. Profiles of topographic distribution and severity of spongiform degeneration in the brains of bank voles harboring T1-T3 phenotypes after inoculation with brain homogenate from variably protease sensitive prionopathy (VPSPr) and control bank voles inoculated with SCJD. A) Spongiform degeneration characterized both T1 and T2 phenotypes but displayed significantly divergent distributions in 5 of the 10 anatomic locations examined; spongiform degeneration affected primarily the cerebral cortex in T1 and the hypothalamus and brain stem in T2; no difference in vacuolar mean diameter was observed between T1 and T2. Spongiform degeneration scores associated with the T3 phenotype were minimal or absent in most locations except for the white matter, especially in the corpus callosum, which was virtually unaffected in T1 and T2. ${ }^{*} p<0.5 ;{ }^{* *} p<0.006 ;{ }^{* *} p<0.0001$ of T1 versus T2 and T3 WM versus T1 and T2; inocula: T1 and T2 VPSPr-129MV, T3 VPSPr-129MM; vacuoles measured $(n=\approx 2,000)$ in T1 and T2 combined. B) Comparative study of T1 profiles generated in bv109M and bv109I revealed an overall more severe spongiform degeneration in bv109I but no significant difference in distribution ( $\dagger, p<0.001, \ddagger, p<0.003 ; N=3$ Bv109I and Bv109M). The T1 spongiform degeneration profile generated by bv109M after inoculation with VPSPr-129MM reproduced the profile generated with sCJDMM1 extracts used as control for human type 1 (bv109M N = 3 for each profile). Similar results were obtained when comparing the T1 profile of bv109I inoculated with VPSPr-129MV and profiles of bv109I inoculated with sCJDMV1 (data not shown). BN, basal nuclei; BSs and BSi, brainstem superior and inferior; bv, bank vole; CC, cerebral cortex; Ce, cerebellum; ctrl, control; Hi, hippocampus; Hth, hypothalamus; sCJD, sporadic Creutzfeldt-Jakob disease; Sept.N, septal nuclei; Th, thalamus; WM, white matter.

anatomic regions such as neocortex and hippocampus, thalamus, and superior colliculi (Figure 3). The T3 phenotype was characterized by the striking presence of $\mathrm{PrP}^{\mathrm{D}}$ in hippocampus and white matter structures (Figure 3).

\section{PrPD Characterization}

Immunoblot analysis confirmed the presence of 3 distinct resPrP $\mathrm{P}^{\mathrm{D}}$ electrophoretic profiles that matched the 3 histopathologic phenotypes. When probed with antibodies 9A2 and $12 \mathrm{~B} 2$, resPrPD $\mathrm{P}^{\mathrm{D}}$ associated with the $\mathrm{T} 1$ phenotype populated 3 bands of $\approx 32,26$, and $21 \mathrm{kDa}$, representing the 3 resPrP $\mathrm{P}^{\mathrm{D}}$ glycoforms, and by a fragment of $7 \mathrm{kDa}$ (Figure 4). An additional C-terminal fragment of $\approx 13 \mathrm{kDa}$, possibly homologous to the human C-terminal fragment 12/13 (20), was detected by the C-terminal antibody SAF84 (Figure 4). Glycoform ratios showed a comparable representation of the diglycosylated and monoglycosylated forms of resPrP $\mathrm{P}^{\mathrm{D}}$ (Figure 5; Appendix Figure 2). The electrophoretic profile and glycoform ratios of resPrP $\mathrm{P}^{\mathrm{D}} \mathrm{T} 1$ conformer were indistinguishable from those of resPrP $\mathrm{P}^{\mathrm{D}}$ observed in bank voles inoculated with SCJDMM1 or SCJDMV1 prions, used as controls for human resPrP type 1 in bank voles (Figures 4, 5; Appendix Figure 2; data not shown).

The resPrPD profile associated with the $\mathrm{T} 2$ phenotype showed 3 bands of $\approx 30,24$, and $19 \mathrm{kDa}$ (i.e., all that had an $\approx 2-\mathrm{kDa}$ faster electrophoretic mobility than the corresponding bands of resPrPD $\mathrm{T} 1$ ) (Figure 4). The 7-kDa fragment was not detected in T2 (Figure 4). In contrast to T1, the T2 glycoform ratio was characterized by the unambiguous predominance of the monoglycosylated component (Figure 5). In summary, bank vole resPrP $\mathrm{P}^{\mathrm{D}} \mathrm{T} 2$ differed from the $\mathrm{T} 1$ conformer by overall 2-kDa faster mobility, the absence of the $7-\mathrm{kDa}$ fragment, and marked predominance of the monoglycoform. The striking feature of the resPrPD associated with the $\mathrm{T} 3$ phenotype was the predominant presence of the $7-\mathrm{kDa}$ fragment detected by $9 \mathrm{~A} 2$ and $12 \mathrm{~B} 2$ but not by SAF84, demonstrating its internal origin and the absence of glycosylation sites (Figure 4).

Additional divergent features emerged when amounts of totPrP $\mathrm{P}^{\mathrm{D}}$ (i.e., PK-sensitive plus resPrP $\mathrm{P}^{\mathrm{D}}$ fractions) were assessed as percentages of total $\mathrm{PrP}$, comprising $\mathrm{PrP}^{\mathrm{C}}$ and totPrP ${ }^{\mathrm{D}}$ (Figure 6). A significantly larger component of totPrP $\mathrm{P}^{\mathrm{D}}$ was resPrP $\mathrm{P}^{\mathrm{D}}$ in $\mathrm{T} 1$ than in $\mathrm{T} 2(81 \%$ vs. $33 \%)$; tot $\operatorname{Pr} \mathrm{P}^{\mathrm{D}}$ fractions were similar $(93 \%$ for $\mathrm{T} 1,91 \%$ for T2). T3 differed significantly: totPrPD accounted for $8 \%$ and $\operatorname{res} \operatorname{PrP}^{\mathrm{D}}$ accounted for $0.2 \%$ of total $\operatorname{PrP}$ (Figure 6; Appendix Figure 3).

\section{Discussion}

The permissiveness of bank vole $\operatorname{PrP}^{\mathrm{C}}$ is well known $(15,16,18,21-27)$; it is exemplified by the observation that, despite the mere 8-aa $\operatorname{PrP}^{\mathrm{C}}$ divergence between bank voles and mice, a variety of human and animal prion diseases not transmissible to mice are infectious to bank voles and 
A $\mathrm{T} 1$

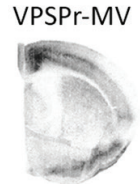

Type 1

SCJDMV1

control
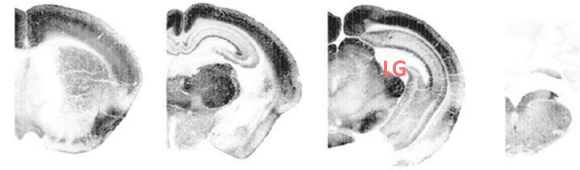

$\mathrm{T} 2$
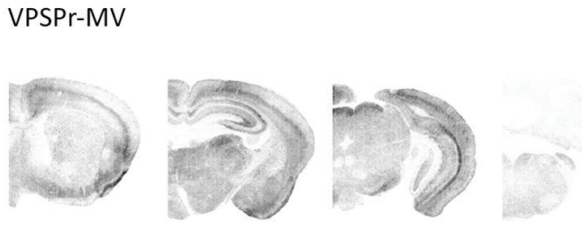

T3

VPSPr-MM
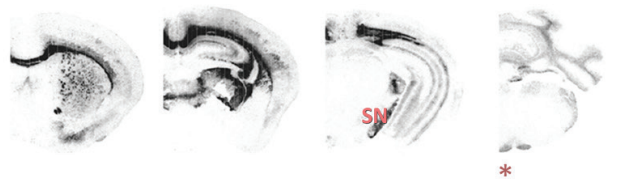

$\mathrm{T} 1$
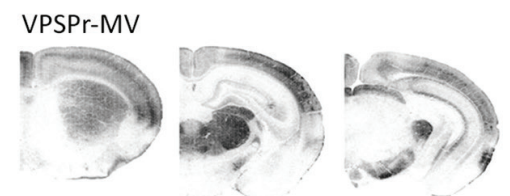

Type 1 sCJDMV1 control
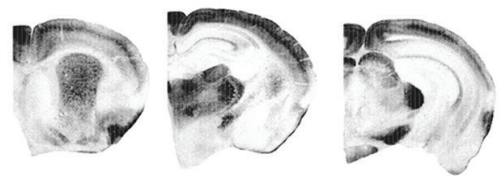

Figure 3. Representative paraffin-embedded tissue (PET) blots of protease-resistant, disease-related prion protein (resPrP $)$ distribution in phenotypes $\mathrm{T} 1-\mathrm{T} 3$ and controls. A) For $\mathrm{T} 1, \mathrm{PrP}^{\mathrm{D}}$ predominated in cerebral cortex $(C)$, thalamus $(T)$, superior colliculus $(S C)$, lateral geniculate nucleus (LG), and substantia nigra (SN). A similar PrPD distribution was observed with transmission of SCJDMV1 used as type control. T2 showed a more uniform distribution in cerebral cortex and subcortical nuclei of an apparently lesser amount of PrPD; T3 appeared to preferentially affect the hemispheric white matter and other subcortical regions such as the alveus, the corpus callosum, the anterior commissure, and fascicles surrounding thalamus as well as other white matter formations such as fimbria, brachium of superior colliculus, medial lemniscus, and cerebral peduncles. Small amounts of $\operatorname{PrP}^{D}$ were also observed in cerebellar and medullary white matter (asterisk [ $\left.{ }^{*}\right]$ ). B) PrPD T1 distribution resembled that of bank voles (bv) 109I after transmission of the same VPSPr-MV brain homogenate (compare with $\mathrm{T} 1$ in $\mathrm{A}$ ). A similar distribution was also observed after inoculation with SCJDMV1. Left to right: coronal sections of telencephalon midlevel caudate nucleus; diencephalon midlevel thalamus; midbrain; and hindbrain-level medulla and cerebellum. SCJD, sporadic Creutzfeldt-Jakob disease; VPSPr, variably protease-sensitive prionopathy. transgenic mice expressing bank vole $\operatorname{PrP}(15,16,18,22,24)$. Conversely, the 15 -aa difference from the human $\operatorname{PrP}^{\mathrm{C}}$ primary sequence does not impede the efficient transmission of a wealth of sporadic and inherited human prion diseases $(15,16)$. This striking permissiveness has been attributed to the presence of several asparagine and glutamine residues in and around the $\beta 2-\alpha 2$ loop that would result in a $\mathrm{PrP}^{\mathrm{C}}$ conformation compatible with the conformations of a large number of $\operatorname{PrP}^{\mathrm{D}}$ strains (21). Furthermore, the polymorphism at bank vole codon 109 adds further complexity to the interaction with exogenous strains $(18,28)$.

We undertook systematic transmission of VPSPr brain homogenates to bv109M and bv109I after failure to consistently transmit VPSPr to humanized transgenic mice. Overall, transmission was favored by the 109I genotype, which propagated all 3 VPSPr 129 genotypes while bv109M failed to transmit VPSPr-VV. However, at first passage in bv109I, the mean attack rate (35\%) was fairly low and the mean survival time ( $575 \mathrm{dpi}$ ) quite extended (Table 1). These conditions changed at second passage, when the attack rate became $100 \%$ in all transmission experiments and survival times decreased on average by $53 \%$ (Table 1 ). These findings point to the existence of a substantial barrier at first passage, which, judging from the $100 \%$ attack rate, is probably largely diminished or vanished at second passage. In view of the aforementioned easy transmissibility of other human prion diseases, the barrier appears to be conformational rather than caused by species-related variations in amino acid sequence of $\operatorname{PrP}^{\mathrm{C}}(15,16)$; the barrier might be associated with the misfolding of VPSPr PrPD , which may be peculiar because after PK digestion it results in an array of highly heterogeneous fragments and apparently the failure to convert one of the glycoforms $(6,7)$. Similarly, the clear effect of the genotype at codon 129 on the attack rate (which was 3-4 times lower for bank voles inoculated with VPSPrVV prions compared with VPSPr-MM and -MV), along with the lack of transmission of VPSPr-VV to bv109M but not to bv109I, points to conformational differences between $\operatorname{PrP}^{\mathrm{D}}$ species associated with the 129 genotypes in VPSPr (16). This notion is further supported by previous data showing higher PK sensitivity (7) and conformational stability of PrP (29) in VPSPr-VV compared with VPSPr-MM and -MV.

The comparative study of VPSPr bioassay in bank voles and humanized transgenic mice revealed substantial differences. VPSPr-challenged mice invariably remained asymptomatic, and all histologically positive mice failed to transmit at second passage. Furthermore, the VPSPrMV subtype was never transmitted to mice $129 \mathrm{M}$ or $129 \mathrm{~V}$, and the general attack rate (assessed histopathologically) was low (54\%); resPrPD was demonstrated in only $34 \%$ of the challenged mice despite the 2-8 times normal levels of PrP expression for most mice (13). However, in contrast to bank voles, positive mice generated a resPrP ${ }^{\mathrm{D}}$ conformer 
Figure 4. Immunoblot characteristics of protease-resistant, disease-related prion protein $\left(\right.$ resPrP ${ }^{\triangleright}$ ) distribution in phenotypes T1-T3 and controls. Regular and long exposures revealed the overall similarity of the 3-band profiles in $\mathrm{T} 1$ and $\mathrm{T} 2$, but resPrPD profile, including glycoform representation, differed in the 2 phenotypes with all 3 monoclonal antibodies (Ab) used. T1 included a 7-kDa band, not detected in T2, similar to mobility and $\mathrm{Ab}$ immunoreactivity of the T3 7-kDa fragment. The T1 profile matched the profile generated in isogenic bank voles inoculated with sCJDMV1 used as human resPrPD type 1 control (ctrl) (lane 2). The T3 profile, visible only after long film exposures, featured a 7-kDa band, but slower migrating bands with variable immunoreactivity were also visible. None of the T1-T3 profiles matched the original VPSPr profile (first lane) although the $\approx 7$ $\mathrm{kDa}$ and both 23-kDa and 19-kDa bands were shared with $\mathrm{T} 1$ and $\mathrm{T} 2$, respectively (compare first with $\mathrm{T} 1$ and T2 lanes). The complexity of the native resPrPD profile from VPSPr homogenate is demonstrated by probing with 1E4, a monoclonal Ab to human PrP highly reactive to VPSPr resPrPD (top right panel) (6). Monoclonal Ab 12B2 (middle panels) with high affinity for human resPrPD type 1 confirmed the type 1 characteristics of the resPrPD associated with the $\mathrm{T} 1$ phenotype. The small amount of resPrP ${ }^{\mathrm{D}}$ type 1 in $1 \mathrm{~T} 2$ bank vole probably represents incomplete proteinase $\mathrm{K}$ (PK) digestion (lane 5, right panel) (19). Monoclonal Ab SAF84 to the PrP C-terminus, unreactive to human PrP, further underlined the divergence in resPrPD primary structure in T1 and T2 compared with T3. Aside from revealing an additional 13-kDa fragment, strongly detected in T1 and T2 and weakly in T3, SAF84 did not detect the 7-kDa fragment, supporting its internal origin (i.e., cleaved at both N- and C-termini). Uninoculated bank voles were negative for resPrPD. All samples were PK treated. sCJD, sporadic Creutzfeldt-Jakob disease; uninoc., not inoculated; polym., polymorphism; VPSPr, variably protease-sensitive prionopathy. very similar to that of VPSPr for electrophoretic profile, glycosylation pattern, and antibody immunoreactivity, although it exhibited higher protease resistance.

Data from a previous study of transmission to humanized transgenic mice and bv109M of an SCJDMV variant with an atypical glycoform profile (CJD-MV ${ }^{\mathrm{AG}}$ ) partially resembled ours (17). Challenged transgenic mice remained asymptomatic and negative at neuropathologic examination, but $22 \%$ of them reproduced the original resPrP $\mathrm{P}^{\mathrm{D}}$ electrophoretic profile and glycotype of the inoculum. In contrast to humanized transgenic mice, bank voles had full-blown disease develop featuring 3 , although partially merging, histopathologic phenotypes along with 3 distinct resPrP $\mathrm{P}^{\mathrm{D}}$ conformers, none of which mimicked the profile and glycotype of the inoculum (17). Remarkably, the glycoform variation of SCJDMV ${ }^{\mathrm{AG}}$ resembles that of VPSPr because both resPrPD species lack the diglycosylated isoform, implicating this variation as one of the possible causes of bank vole failure to accurately replicate exogenous $\operatorname{PrP}^{\mathrm{D}}$ (17).
Three subtypes of GSS (which VPSPr resembles in terms of the ladder-like electrophoretic profile and the sensitivity to $\mathrm{PK}$ of resPrP $\mathrm{P}^{\mathrm{D}}$ ) have also recently been transmitted to bank voles and 1 GSS subtype to humanized transgenic mice $(16,30)$. Despite the well-known difficulty of transmitting GSS to rodents, bank voles challenged with 2 major GSS subtypes associated with PrP mutations A117V and F198S (GSS ${ }^{\mathrm{A1} 17 \mathrm{~V}}, \mathrm{GSS}^{\mathrm{F} 198 \mathrm{~S}}$ ) showed no evidence of species or mutation barrier. Transmission was comparatively more difficult with the third GSS ${ }^{\text {P102L }}$ subtype, in which resPrP $\mathrm{P}^{\mathrm{D}}$ displays 2 sets of fragments: either the $8-\mathrm{kDa}$ fragment associated with the $30-21 \mathrm{kDa}$ glycoform triplet $(31,32)$ or the $8-\mathrm{kDa}$ fragment alone. After inoculation, the 2-fragment set was never replicated, and the $\approx 8-\mathrm{kDa}$ fragment alone occasionally was inaccurately reproduced as a $7-\mathrm{kDa}$ fragment $(16,28)$. To date, only $\mathrm{GSS}^{\mathrm{A117v}}$ has been transmitted to 2 lines of transgenic mice expressing human $\operatorname{PrP}^{\mathrm{D}}$ harboring the $\mathrm{A} 117 \mathrm{~V}$ transition (30). Although transmission features diverged in the 2 lines, both seemed 
to reproduce the 7-kDa fragment that is the only strongly resPrP ${ }^{\mathrm{D}}$ fragment in this disease.

Combined, these experiments indicate that $\mathrm{PrP}^{\mathrm{C}}$ characteristics, and possibly other host factors (25), enable bank voles to be more permissive hosts (despite the species barrier) than transgenic mice expressing conspecific $\operatorname{PrP}^{\mathrm{C}}$, confirming the empirical aspect of the species barrier. However, bank vole $\mathrm{PrP}^{\mathrm{C}}$ can hardly reproduce faithfully complex features of human atypical prion isolates, a task that may require $\mathrm{PrP}^{\mathrm{C}}$ from the same species.

A remarkable finding of this study is the occurrence of 3 well-defined histopathologic phenotypes (T1-T3), which displayed discrete $\operatorname{PrP}^{\mathrm{D}}$ brain distribution and were linked to $\operatorname{PrP}^{\mathrm{D}}$ conformers easily distinguishable by electrophoretic profile and glycosylation characteristics. The 3 phenotypes also differed by mean survival times at first and second passages. Remarkably, the T1-T3 phenotypes were often generated by the same inoculum but never co-occurred in the same bank vole. Combined, these features define the T1-T3 $\operatorname{PrP}^{\mathrm{D}}$ conformers as distinct strains, raising the issue of their origin. Both histopathologic and resPrP $\mathrm{P}^{\mathrm{D}}$ electrophoretic characteristics of the $\mathrm{T} 1$ phenotype are essentially indistinguishable from those of bank voles inoculated with sCJDMV1. Data on transmission of sCJDMM2, available only for bv109M, show that the electrophoretic profile of the newly formed resPrP ${ }^{\mathrm{D}}$ matches the $\mathrm{T} 2$ resPrP $\mathrm{P}^{\mathrm{D}}$ of this study (15). Although the $\mathrm{T} 1$ and $\mathrm{T} 2$ representations of totPrP $\mathrm{P}^{\mathrm{D}}$ and $\operatorname{resPrP} \mathrm{P}^{\mathrm{D}}$ are not known in bank voles inoculated with sCJDMM1 and sCJDMM2 prions, the values we observed after VPSPr

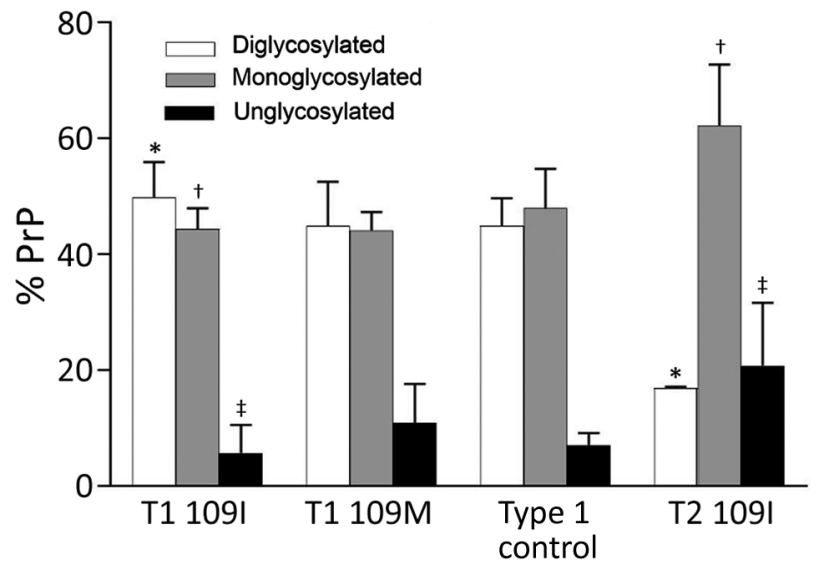

Figure 5. Glycoform ratio of protease-resistant, disease-related prion protein $\left(\right.$ resPrP $\left.{ }^{D}\right)$ in phenotypes $\mathrm{T} 1$ and $\mathrm{T} 2$. The ratio of resPrPD associated with T1 (T1 109I) was $48 \%$ for diglycosylated, $44 \%$ for monoglycosylated, and $8 \%$ for unglycosylated conformers and significantly differed in each glycoform from the $17 \%$, $63 \%$, and $20 \%$ corresponding ratio of T2 (T2 109I). ${ }^{*} p<0.0001$; $\dagger p<0.005 ; \ddagger p<0.05$. Glycoform ratios of T1 109 I and T1 109M as well as that of type 1 control (from bank voles 109 I inoculated with sporadic Creutzfeldt-Jakob disease MV1 and used as human type 1 controls) did not significantly differ from each other. Each bar represents mean \pm SD of $n=4$ for T1 109M, $n=6$ for T1 109l, $\mathrm{n}=6$ for $\mathrm{T} 2$, and $\mathrm{n}=2$ for type 1 control.

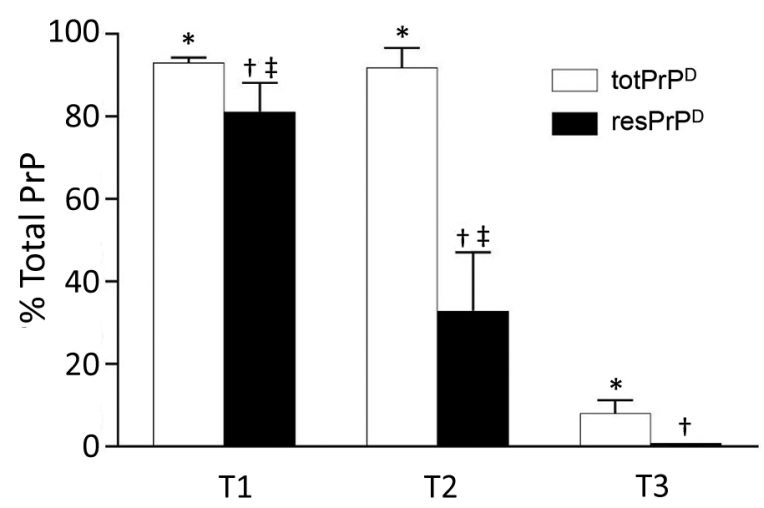

Figure 6. Relative quantities of totPrPD and resPrPD in T1-T3 phenotypes. totPrPD accounted for $93.1 \%$ and resPrPD for $81.3 \%$ of total PrP recovered from bank voles harboring the T1 phenotype. Corresponding percentages for $\mathrm{T} 2$ were $91.0 \%$ and $33.0 \%$, and T3 totPrPD and resPrPD accounted only for $8.0 \%$ and $0.2 \%$ of total PrP and differed significantly from both $\mathrm{T} 1$ and $\mathrm{T} 2$ in each of the 2 components. ResPrPD also differed significantly between $\mathrm{T} 1$ and T2 (each bar represents the mean \pm SD of $n=3 T 1, n=3$ T2, and n = 5 T3; all data are from bank voles 109l; antibody 9A2). bv, bank vole; resPrPD protease-resistant, disease-related prion protein; totPrPD, comprising protease-sensitive PrPD and resPrPD. ${ }^{*} p<0.0001 ; \uparrow p<0.0001$ vs. T1 and $p<0.05$ vs. T2; $\neq p<0.01$.

inoculation are comparable to those reported for the original sCJD, in which totPrP $\mathrm{P}^{\mathrm{D}}$ and resPrP $\mathrm{P}^{\mathrm{D}}$ reportedly accounted for $53.5 \%$ and $48.2 \%$ of total PrP in SCJDMM1 (6; L. Cracco et al., unpub. data). Therefore, transmission to bank voles suggests that VPSPr $\operatorname{PrP}^{\mathrm{D}} \mathrm{T} 1$ and $\mathrm{T} 2$ are related to human $\operatorname{PrP}^{\mathrm{D}}$ types 1 and 2, respectively. In contrast, phenotype T3 is the most divergent, especially for spongiform degeneration and $\operatorname{PrP}^{\mathrm{D}}$ deposition, mostly limited to white matter regions, and electrophoretic profile, where resPrP $\mathrm{P}^{\mathrm{D}}$ recovered as a band of $7 \mathrm{kDa}$, was the major component shared with the complex pattern of VPSPr resPrP ${ }^{\mathrm{D}}$. The T3 histopathologic phenotypes including the PrP immunostaining pattern matched also the bank vole phenotype of GSS ${ }^{\mathrm{A} 117 \mathrm{~V}}$ and $\mathrm{GSS}^{\mathrm{P} 102 \mathrm{~L}}$ associated with the 8-kDa fragment only (16). The exceedingly low representation of the totPrP ${ }^{\mathrm{D}}$ and $\operatorname{resPrP}^{\mathrm{D}}$ components of total PrP in T3 is reminiscent of the corresponding data reported in VPSPr-VV, in which totPrP $\mathrm{P}^{\mathrm{D}}$ accounted for $3.4 \%$ and resPrP $\mathrm{P}^{\mathrm{D}}$ for $0.83 \%$ of total $\operatorname{PrP}(6)$. The marked underrepresentation of totPrPD and resPrP $\mathrm{PD}^{\mathrm{D}}$ in $\mathrm{T} 3$ is especially puzzling considering that attack rate and survival time are not very different from those of $\mathrm{T} 2$ and $\mathrm{T} 1$, respectively. The apparent relative high efficiency of $\mathrm{T} 3$ might be explained by the high representation of oligomers (33). Alternatively, the $\mathrm{T} 3$ underrepresentation of totPrP ${ }^{\mathrm{D}}$ relative to total PrP might reflect the lack of $\operatorname{PrP}^{\mathrm{C}}$ down-regulation by $\mathrm{T} 3$ compared with $\mathrm{T} 1$ and $\mathrm{T} 2$, which would result in the relative increase of the total PrP pool $(33,34)$.

A mechanism put forward for the lack of fidelity in cross-species transmission of the prion strain $(25,35-37)$ is 
based on evidence that the dominant strain is selected from an array of strains that persist as substrains. In cross-species transmissions, substrains may be selected over the dominant strain (38-40). In the context of VPSPr, this mechanism is particularly intriguing, given that small quantities of $\operatorname{PrP}^{\mathrm{D}}$ conformers with electrophoretic mobilities similar to those of human $\operatorname{PrP}^{\mathrm{D}}$ type 1 were originally observed in a few cases by Gambetti et al (6); more recently, the presence of $\operatorname{PrP}^{\mathrm{D}}$ type 2 in VPSPr, mostly in subcortical nuclei and in cerebellum, has been reported $(11,12)$. These 2 components would be propagated faithfully in T1 and T2, and T3, which consistently shares only the 7-kDa fragment with the VPSPr resPrP ${ }^{\mathrm{D}}$, might represent the isolation of this GSSlike component of VPSPr resPrPD or the unsuccessful attempt to fully reproduce the dominant strain associated with this disease. We and others have occasionally observed an underrepresented 7-kDa fragment in SCJDMM1 (41; S. Notari, P. Gambetti, P. Parchi, unpub. data). Thus, it is tempting to speculate that the 7-kDa fragment observed in bank voles inoculated with SCJDMM1 and SCJDMV1 prions is related to the presence and possibly the infectivity of such fragment in the $\operatorname{SCJDMM}(\mathrm{MV}) 1$ subtype.

In conclusion, on the basis of the first full transmission of VPSPr, our study confirms the permissiveness of bank voles to human prion diseases and suggests that bank voles are competent to reveal minor strain variants in prion diseases, such as resPrP ${ }^{\mathrm{D}}$ types 1 and 2 reported in VPSPr and, possibly, the $\approx 7-\mathrm{kDa}$ fragment observed in SCJDMM1 and sCJDMV1. However, our study also underscores the limited competence of bank vole $\mathrm{PrP}^{\mathrm{C}}$ to faithfully reproduce the multiband profile of VPSPr resPrP $P^{\mathrm{D}}$ that probably reflects the complex conformation of the prion seed in this disease.

\section{Acknowledgments}

We thank Janis Blevins, Katie Eppich, Yvonne Cohen, and the other personnel of the National Prion Disease Pathology Surveillance Center as well as Geraldina Riccardi, Stefano Marcon, Paolo Frassanito, and Marcello Rossi for their skillful assistance provided during the course of this study.

This work was supported by the National Institutes of Health (P01AI106705, 5R01NS083687) and the Charles S. Britton Fund (to P.G.).

\section{About the Author}

Dr. Nonno is a research scientist in the Emerging Zoonosis Unit at the Istituto Superiore di Sanità in Rome, Italy; his primary research interests include prion strain characterization and the zoonotic potential of animal prion diseases. Dr. Notari is an instructor in the Department of Pathology at Case Western Reserve University, Cleveland, Ohio, USA; his research focuses mainly on prion diseases, particularly prion molecular characteristics and infectivity.

\section{References}

1. Gambetti P. Creationism and evolutionism in prions. Am J Pathol. 2013;182:623-7. http://dx.doi.org/10.1016/j.ajpath.2012.12.016

2. Gambetti P, Cali I, Notari S, Kong Q, Zou WQ, Surewicz WK. Molecular biology and pathology of prion strains in sporadic human prion diseases. Acta Neuropathol. 2011;121:79-90. http://dx.doi.org/10.1007/s00401-010-0761-3

3. Puoti G, Bizzi A, Forloni G, Safar JG, Tagliavini F, Gambetti P. Sporadic human prion diseases: molecular insights and diagnosis. Lancet Neurol. 2012;11:618-28. http://dx.doi.org/10.1016/ S1474-4422(12)70063-7

4. Parchi P, Strammiello R, Giese A, Kretzschmar H. Phenotypic variability of sporadic human prion disease and its molecular basis: past, present, and future. Acta Neuropathol. 2011;121:91-112. http://dx.doi.org/10.1007/s00401-010-0779-6

5. Poggiolini I, Saverioni D, Parchi P. Prion protein misfolding, strains, and neurotoxicity: an update from studies on mammalian prions. Int J Cell Biol. 2013;2013:910314. http://dx.doi.org/ $10.1155 / 2013 / 910314$

6. Gambetti P, Dong Z, Yuan J, Xiao X, Zheng M, Alshekhlee A, et al. A novel human disease with abnormal prion protein sensitive to protease. Ann Neurol. 2008;63:697-708. http://dx.doi.org/10.1002/ana.21420

7. Zou WQ, Puoti G, Xiao X, Yuan J, Qing L, Cali I, et al. Variably protease-sensitive prionopathy: a new sporadic disease of the prion protein. Ann Neurol. 2010;68:162-72. http://dx.doi.org/10.1002/ ana.22094

8. Notari S, Appleby B, Gambetti P. Variably protease sensitive prionopathy. In: Pocchiari M, Manson J. editors. Handbook of clinical neurology, vol. 153. San Diego: Elsevier; 2018. p. 175-190.

9. Collins SJ, Sanchez-Juan P, Masters CL, Klug GM, van Duijn C, Poleggi A, et al. Determinants of diagnostic investigation sensitivities across the clinical spectrum of sporadic CreutzfeldtJakob disease. Brain. 2006;129:2278-87. http://dx.doi.org/10.1093/ brain/awl159

10. Pirisinu L, Nonno R, Esposito E, Benestad SL, Gambetti P, Agrimi U, et al. Small ruminant nor98 prions share biochemical features with human Gerstmann-Sträussler-Scheinker disease and variably protease-sensitive prionopathy. PLoS One. 2013;8:e66405. http://dx.doi.org/10.1371/journal.pone.0066405

11. Rodríguez-Martínez AB, López de Munain A, Ferrer I, Zarranz JJ, Atarés B, Villagra NT, et al. Coexistence of protease sensitive and resistant prion protein in 129VV homozygous sporadic CreutzfeldtJakob disease: a case report. J Med Case Reports. 2012;6:348. http://dx.doi.org/10.1186/1752-1947-6-348

12. Peden AH, Sarode DP, Mulholland CR, Barria MA, Ritchie DL, Ironside JW, et al. The prion protein protease sensitivity, stability and seeding activity in variably protease sensitive prionopathy brain tissue suggests molecular overlaps with sporadic Creutzfeldt-Jakob disease. Acta Neuropathol Commun. 2014;2:152. http://dx.doi.org/10.1186/s40478-014-0152-4

13. Notari S, Xiao X, Espinosa JC, Cohen Y, Qing L, Aguilar-Calvo P, et al. Transmission characteristics of variably protease-sensitive prionopathy. Emerg Infect Dis. 2014;20:2006-14. http://dx.doi.org/ 10.3201/eid2012.140548

14. Diack AB, Ritchie DL, Peden AH, Brown D, Boyle A, Morabito L, et al. Variably protease-sensitive prionopathy, a unique prion variant with inefficient transmission properties. Emerg Infect Dis. 2014;20:1969-79. http://dx.doi.org/10.3201/eid2012.140214

15. Nonno R, Angelo Di Bari M, Agrimi U, Pirisinu L. Transmissibility of Gerstmann-Sträussler-Scheinker syndrome in rodent models: new insights into the molecular underpinnings of prion infectivity. Prion. 2016;10:421-33. http://dx.doi.org/10.1080/ 19336896.2016.1239686

16. Pirisinu L, Di Bari MA, D’Agostino C, Marcon S, Riccardi G, Poleggi A, et al. Gerstmann-Sträussler-Scheinker disease subtypes 
efficiently transmit in bank voles as genuine prion diseases. Sci Rep. 2016;6:20443. http://dx.doi.org/10.1038/srep20443

17. Galeno R, Di Bari MA, Nonno R, Cardone F, Sbriccoli M, Graziano S, et al. Prion strain characterization of a novel subtype of Creutzfeldt-Jakob disease. J Virol. 2017;91:e2390-16. http://dx.doi.org/10.1128/JVI.02390-16

18. Watts JC, Giles K, Patel S, Oehler A, DeArmond SJ, Prusiner SB. Evidence that bank vole PrP is a universal acceptor for prions. PLoS Pathog. 2014;10:e1003990. http://dx.doi.org/10.1371/ journal.ppat. 1003990

19. Notari S, Capellari S, Langeveld J, Giese A, Strammiello R, Gambetti P, et al. A refined method for molecular typing reveals that co-occurrence of $\operatorname{PrP}(\mathrm{Sc})$ types in Creutzfeldt-Jakob disease is not the rule. Lab Invest. 2007;11:1103-12.

20. Zou WQ, Capellari S, Parchi P, Sy MS, Gambetti P, Chen SG. Identification of novel proteinase $\mathrm{K}$-resistant $\mathrm{C}$-terminal fragments of PrP in Creutzfeldt-Jakob disease. J Biol Chem. 2003;278: 40429-36. http://dx.doi.org/10.1074/jbc.M308550200

21. Agrimi U, Nonno R, Dell'Omo G, Di Bari MA, Conte M, Chiappini B, et al. Prion protein amino acid determinants of differential susceptibility and molecular feature of prion strains in mice and voles. PLoS Pathog. 2008;4:e1000113. http://dx.doi.org/ 10.1371/journal.ppat.1000113

22. Di Bari MA, Chianini F, Vaccari G, Esposito E, Conte M, Eaton SL, et al. The bank vole (Myodes glareolus) as a sensitive bioassay for sheep scrapie. J Gen Virol. 2008;89:2975-85. http://dx.doi.org/10.1099/vir.0.2008/005520-0

23. Cosseddu GM, Nonno R, Vaccari G, Bucalossi C, Fernandez-Borges N, Di Bari MA, et al. Ultra-efficient PrPSc amplification highlights potentialities and pitfalls of PMCA technology. PLoS Pathog. 2011;7:e1002370. http://dx.doi.org/ 10.1371/journal.ppat.1002370 PMID: 22114554

24. Di Bari MA, Nonno R, Castilla J, D’Agostino C, Pirisinu L, Riccardi G, et al. Chronic wasting disease in bank voles: characterisation of the shortest incubation time model for prion diseases. PLoS Pathog. 2013;9:e1003219. http://dx.doi.org/ 10.1371/journal.ppat.1003219

25. Espinosa JC, Nonno R, Di Bari M, Aguilar-Calvo P, Pirisinu L, Fernández-Borges N, et al. PrPC governs susceptibility to prion strains in bank vole, while other host factors modulate strain features. J Virol. 2016;90:10660-9. http://dx.doi.org/10.1128/ JVI.01592-16

26. Orrú CD, Groveman BR, Raymond LD, Hughson AG, Nonno R, Zou W, et al. Bank vole prion protein as an apparently universal substrate for RT-QuIC-based detection and discrimination of prion strains. PLoS Pathog. 2015;11:e1004983. http://dx.doi.org/10.1371/ journal.ppat.1004983

27. Fernández-Borges N, Eraña H, Castilla J. Behind the potential evolution towards prion resistant species. Prion. 2018;12:83-7. http://dx.doi.org/10.1080/19336896.2018.1435935

28. Nonno R, Di Bari MA, Cardone F, Vaccari G, Fazzi P, Dell'Omo G, et al. Efficient transmission and characterization of Creutzfeldt-Jakob disease strains in bank voles. PLoS Pathog. 2006;2:e12. http://dx.doi.org/10.1371/journal.ppat.0020012

29. Pirisinu L, Marcon S, Di Bari MA, D’Agostino C, Agrimi U, Nonno R. Biochemical characterization of prion strains in bank voles. Pathogens. 2013;2:446-56. http://dx.doi.org/10.3390/ pathogens 2030446

30. Asante EA, Linehan JM, Smidak M, Tomlinson A, Grimshaw A, Jeelani A, et al. Inherited prion disease A117V is not simply a proteinopathy but produces prions transmissible to transgenic mice expressing homologous prion protein. PLoS Pathog. 2013;9:e1003643. http://dx.doi.org/10.1371/journal.ppat. 1003643

31. Parchi P, Chen SG, Brown P, Zou W, Capellari S, Budka H, et al. Different patterns of truncated prion protein fragments correlate with distinct phenotypes in P102L Gerstmann-SträusslerScheinker disease. Proc Natl Acad Sci U S A. 1998;95:8322-7. http://dx.doi.org/10.1073/pnas.95.14.8322

32. Piccardo P, Manson JC, King D, Ghetti B, Barron RM. Accumulation of prion protein in the brain that is not associated with transmissible disease. Proc Natl Acad Sci U S A. 2007;104:4712-7. http://dx.doi.org/10.1073/pnas.0609241104

33. Mays CE, Kim C, Haldiman T, van der Merwe J, Lau A, Yang J, et al. Prion disease tempo determined by host-dependent substrate reduction. J Clin Invest. 2014;124:847-58. http://dx.doi.org/10.1172/JCI72241

34. Mays CE, van der Merwe J, Kim C, Haldiman T, McKenzie D, Safar JG, et al. Prion infectivity plateaus and conversion to symptomatic disease originate from falling precursor levels and increased levels of oligomeric PrPSc species. J Virol. 2015;89:12418-26. http://dx.doi.org/10.1128/JVI.02142-15

35. Weissmann C. Mutation and selection of prions. PLoS Pathog. 2012;8:e1002582. http://dx.doi.org/10.1371/ journal.ppat. 1002582

36. Makarava N, Savtchenko R, Alexeeva I, Rohwer RG, Baskakov IV. New molecular insight into mechanism of evolution of mammalian synthetic prions. Am J Pathol. 2016;186:1006-14. http://dx.doi.org/ 10.1016/j.ajpath.2015.11.013

37. Moreno JA, Telling GC. Insights into mechanisms of transmission and pathogenesis from transgenic mouse models of prion diseases. Methods Mol Biol. 2017;1658:219-52. http://dx.doi.org/ 10.1007/978-1-4939-7244-9 16

38. Weissmann C, Li J, Mahal SP, Browning S. Prions on the move. EMBO Rep. 2011;12:1109-17. http://dx.doi.org/10.1038/ embor.2011.192

39. Collinge J, Clarke AR. A general model of prion strains and their pathogenicity. Science. 2007;318:930-6. http://dx.doi.org/10.1126/ science. 1138718

40. Li J, Mahal SP, Demczyk CA, Weissmann C. Mutability of prions. EMBO Rep. 2011;12:1243-50. http://dx.doi.org/10.1038/ embor.2011.191

41. Benedetti D, Fiorini M, Cracco L, Ferrari S, Capucci L, Brocchi E, et al. Molecular characterization of low molecular mass C-terminal fragments in different Creutzfeldt-Jakob disease subtypes. Presented at: Prion 2008; 2008 Oct 8-10; Madrid, Spain.

Address for correspondence: Pierluigi Gambetti, Case Western Reserve University, 2085 Adelbert Rd, Rm 419, Cleveland, OH 44160, USA; email: pxg13@case.edu; Umberto Agrimi, Istituto Superiore di Sanità, Viale Regina Elena 299, Rome 00161, Italy; email: umberto.agrimi@iss.it 